\title{
The Clinical Nurse Educator as Inside Researcher
}

*Mr. Allan Seraj, RN

\section{My Position as a Clinical Nurse Educator}

Insider research has been utilized in qualitative research where professionals are seeking to study groups to which they belong. This definition has been substantiated by Breen(2007).

The evolution of the Clinical Nurse Educators' (CNE) role in clinical practice has augmented the way research is conducted within clinical environments. Clinical Nurse Educators' are well placed to advance the way educational research in clinical practice is conducted. As researchers in their own clinical settings, they are challenged to examine the issues associated with studying a group to which they belong. They become endogenous or insiderresearchers. Nurses in clinical practice, according to Tolson et al (2006), are best situated to drive forward an insider based research agenda and engage in the research process. And as an educator, Roberts (2007) argued that we are linked to our learners.

Therefore, as a Clinical Nurse Educators', being a part of the fabric of my clinical learning environment, and being actively involved in the change processes brought about by the everchanging evidence base, I am well placed to conduct research within my work setting.

This however has not been the case, as the use of insider research, according to Brannick \& Coghlan (2007), has not been a widely reported approach for researching clinical settings. Although there have been a lot of criticisms for the use of insider research, in the last decade, many articles have been cited which point to the use of insider research as a valid method of researching groups to which the researcher belongs. There are many advantages of insider research and these are now discussed.

\section{Advantages of Insider Research}

There are three main advantages of being an insider researcher that have been identified by Bonner and Tolhurst (2002) (a) having an established intimacy: this promotes both the telling and the judging of truth: knowing first hand about the research site, its participants and the environment provides a richness to the data collectionand subsequent analysis. This also enhances the outcomes and relevance of the study to that environment. As a philosophical aspect of qualitative research, the researcher must underscore the truth that is being sought, where that truth is situated, how it is derived and its impact on the process (b) having a greater understanding of the culture being studied: this understanding augments, not only data analysis, but how the research is situated in the environment as it affords the researcher to reach into the core of the research group/environment. (c) not altering the flow of social interaction unnaturally: insider research allows for the participants to stay in their 'native environment' and ultimately this allows the data to flow more naturally.

Also, according to Smyth \& Holian (2008) insider-researchers generally know the politics

\section{*Clinical Nurse Educator/Professional Doctorate Student, Bucks NewUni, England, United Kingdom.}


of the institution and how the system works. They are best placed to engage participants and their knowledge of the institution is invaluable compared to someone coming from the outside.Other advantages of insider researching have been noted by Hockey (1993) which include (a) being in a familiar setting and feeling comfortable (b) a lack of culture shock can enhance engagement with participants and communication with participants (c) the inside researcher will have an augmented ability to judge how honest and accurate the responses are because they are part of the institution (d) there is a greater propensity to reflect more deeply with someone who is familiar and sympathetic about their situation thus making for richer data collection I participants' expectations of an insider may be greater as they are both in the same situation-there is the belief that insiders understand the culture and hence share the 'sufferings' of the participants (f) as an insider, you will have better access to naturalistic data and to respondents (g) when using ethnographic approaches, there is potentially the opportunity to produce data that is meaningful to participants (h) because as an insider you understand the culture, you are better able to utilize naturalistic data, critical discourse analysis and phenomenography and (i) insider research is more practical, cheaper and easier.

The use of insider research according to Geertz (1973) empowers the researcher to offer a "thick description of lived realities, of the hermeneutics of everyday life".

Whilst insider research offers a wide range of advantages, there are disadvantages to the method. These are now explored.

\section{Disadvantages of Insider Research}

DeLyser (2001) and Hewitt-Taylor (2002) identified some main disadvantages associated with being an insider researcher: (a) the loss of objectivity as a result of being too familiar with the subject matter. The argument is that being too close to the participant's situation may lead to the loss of the researcher's objectivity about the data (b) Bias can occur as a result of wrong assumptions being made about the research process based on the researcher's prior knowledge.

The phenomenon of role duality, as noted by DeLyser (2001) and Gerrish (1997), can occur with insider research. There is often the struggle to balance the insider's role (instructor, nurse, manager etc.) and the researcher role. They further went on to acknowledge that as an insider, the researcher may not receive or see important information as their judgment may be clouded.

Another risk, as noted by the authors above, may be that the insider researcher gains access to sensitive information. This also becomes an ethical issue as conflict may arise and confidentiality may need to be breached. In these cases, it is advised that the boundaries of confidentiality be made explicit at the start of the data collection.

DeLyser (2001) and Gerrish (1997) warns of the relativity of the insider's status - there is a potential that partiality may arise because of insider knowledge and being wary of assuming their views are more widespread or representative than is the case. They further went on to add that insiders may lose the ability 
to produce good, culturally neutral accounts. There is also the issue of finding it difficult to 'see' some dimensions of social life because they have become normalised in the eyes of the insider researcher. There may also be conflicts between the role as a researcher and your professional role('interview bias').

There is a caution by Holian and Brooks (2004) to insider researchers when they said: "Insider researchers may need to be cautioned and reminded that the research mantle may seem magical and may reveal earth-shattering insights, but it is not bullet proof" (Holian and Brooks 2004:14)

It is acknowledged that there are disadvantages to be considered when attempting to undertake insider research. Another issue that presented during the ethics committee review of my study's proposal was that of power and this is discussed next.

\section{Power Relations in Insider Research}

Power relations in insider research are important in order to fully understand the construct of the method. The insider researcher can, according to (Holian 1999)(a) face unexpected strength of the institution's politics that may hinder the research process. Being part of the system may not necessarily strengthen the insider researcher's position, but, having a greater understanding of the politics may cause the researcher to refrain from engaging wholeheartedly with participants, the data may be superficial or even skewed and bias may present itself (b) there may be abuses of power: researchers who hold positions of authority in the institution may utilize their position to uncover sensitive data and use it against participants. The participants may also feel obliged and potentially coerced to take part in the research project which may lead to poor data collection and bias (c) as an inside researcher with 'inside' knowledge, there is the potential for covering up material that is potentially harmful to the participants' wellbeing, and in the case of clinical practice, patient care (d) the insider researcher may fall prey to rhetoric: there is potential to engage in the research process and then give false promises to participants- they may be swayedby the charisma of the researcher and not fully comprehend the context of the research. This may lead to loss of faith in the researcher, the research process and can reflect negatively on the institution and I there is also the potential that the inside researcher may loose touch with reality: not understanding or overestimating the situation may lead to the researcher loosing touch with the reality of what the data are saying. This can skew the data or even present bias.

Power relations in insider research are crucial in understanding the construct of the method. The use of power in insider research presents with the potential for bias, data skewing and negative impact on the participants and patient care (Shaw 2003)

As I reflected on the effect of power on inside research, I began to examine my role as a Clinical Nurse Educators' and the power dynamics that may present itself as I pursue my study in my own work environment. The use of a reflective diary and the exploration of the ethics surrounding insider research illuminated 
my journey. These augmented my fuller understanding and bridling of power towards enhancing the process.

\section{The Ethics of conducting Insider Research}

Insider researchers are faced with ethical boundaries that they have to overcome in order to fully understand the 'gravity' of their method.

Holian and Brooks (2004) identifies four ethical 'boundaries' of which insider researchers must be considerate (a) the owner of the data and who can 'release' it for research purposes: there is the dilemma that even though the data is collected by the researcher, does it belong to $\mathrm{him} / \mathrm{her}$ or the institution where the research was carried out and is it the sole responsibility of the inside researcher to give permission for dissemination of the findings or the institution? (b) The relationship between the participants and the researcher: if clear boundaries between participants and researcher are not set and respected, the relationship can break down and ultimately the research process (c) insider researchers must consider the nature and level of informed consent: it is crucial that consent is informed and ongoing throughout the research process. Respecting the participant's desire to take part in the research and equally his decision not to continue in the process is important both the researcher and participants and (d) the nature and extent of anonymity and confidentiality for individuals and the organizationis also a vital ethical boundary for the inside researcher: the value of anonymity and confidentiality remains as key ethical principles in the process. The awareness of these principle remains at the forefront of the research process.
Grey (2005) indicates that it is a common understanding that most inside researchers are "likely to be confronted with the dilemma of uncovering information that 'nudges' at ethical boundaries, rather than blatantly transgresses them"

Insider researchers are constantly struggling with the concept of 'normal' and 'acceptable' according to Brannan and Worthington (2007). These writers indicate that subjective attitudes and difficult situations are brought up in the research process that cannot be completely 'bracketed out' and can potentially influence the interpretation of data.The issue of validity, therefore, is an important concept that the inside researcher must consider.

\section{Insider Research Validity}

As an actor within his own setting, insider research has been under scrutiny. The validity of insider research is complicated according to Lesson (2003). The issue of validity is complicated by the relationship that exists between the researcher and the participants. There is the element of subjectivity when it comes to researching your own environment and it is difficult to reference this 'subjectivity'.

There have also been questions raised by Rooney (2005) about the complexities of insider research (a) the researcher's relationship with participants may have a negative impact on the subject's behaviour (b) researcher's biases, may threaten validity and trustworthiness of the research (c) the tacit knowledge of the researcher may lead to the misinterpretation of data or making of false assumptions about the findings (d) prior knowledge of the researcher 
may lead the inside researcher to miss potentially important information I misrepresentation of the whole picture may result from researcher's politics, loyalties, or hidden agenda and (f)data can subconsciously be distorted because of the researcher's moral/political/ cultural standpoint. matters less than a revealing, insightful account and interpretation. Self-reflection is thus crucial.' One can therefore infer that in light of the complex nature of obtaining validity, perhaps it is an objective to be worked towards rather than fully achieving it.

Different views on the validity of insider
Brannick and

Coghlan (2007)

argue that we are all insiders of the society in which we live and that the knowledge we have of these systems we are a part of is deep and contextual.

They further argue that as inside researchers, through reflective awareness, "we develop and utilize

tacit knowledge that become deeply segmented as a result of socialization in an organisational system and reframe it as theoretical knowledge and that because we are close tosomething or know it well, that we can research it.

This, according to Brannick and Coghlan (2007) provides justification for the validity of insider research through recognized methods of reflexivity in line with the appropriate research process.

The idea of self-reflection is supported by Lesson (2003, p. 190) by suggesting 'a more reflective approach in which data management research have been expressed. Lewis (1973) argues that outsiders cannot produce a valuable research

perspective. In contrast, Wolff (1950), believes that the stranger can more easily critically observe events and situations in the research process that an insiders may take for granted as unquestionable "truths".

One proposed resolution of this dilemma according to Maruyama (1991) is to conduct research involving research teams from several 'inside' and/or 'outside' cultures- this however proves to be resource intensive.

Credible insider research, according to Smyth \& Holian (2008), requires awareness of the possible effects of perceived or potential bias on data collection, analysis, consider and address the issues about the influencing researcher's insider role on coercion, respect the ethical issues of anonymity forthe organization and 
participants and compliance and access to privileged information.

'Insiderness' is not a fixed value. Labree (2002) however conceptualises the insider researcher as a fixed point which is contrary to many commentators of inside research who promote it as a continuum-the researcher can go in and out of the situation, both as insider and outsider, depending on the geography within the institution, collecting data from 'colleagues' within the institution but not from your own profession and your substantive position in the institution during the research process.

Merton (1972) suggests that we are never completely an insider researcher and to believe this is a fallacy.

However, as educational research in clinical practice is concerned with human beings and their behaviour, it may be necessary to involve stakeholders, each of who will bring to the research process a wide range of perspectives. In this way, a more balanced situation and 'objective' view can develop, according to May (as cited in Porteli, 2008), thus enhancing the validity of the research process.

\section{Conclusion}

Insider research presents issues of ethical, power relations and research validity to the researcher who opts to study his own working environment. As a Clinical Nurse Educators' choosing to study his own colleagues, it has become apparent that it is crucial to explore all the issues and apply the principles to the research process and the journey. I have learnt that 'insiderness' is a continuum and not a fixed value. As an inside researcher, I must develop awareness of the possible effects of perceived or potential bias on data collection, analysis, consider and address the issues about the influencing researcher's insider role on coercion, respect the ethical issues of anonymity for the organization and participants and compliance and access to privileged information. There is need for wide engagement of stakeholders to balance the potential conflicts associated with insider research. This coupled with reflective awareness will illuminate the researcher's journey and improve the research process.

\section{References}

Brannan, M., Worthington, F. (2007) Ethnographies of work and the work of ethnography. Ethnography; 8: 4, 395-402.

Brannick, T., \& Coghlan, D. (2007). In defense of being native: the case for insider academic research. Organisational Research Methods, 10(1), 59-74.

Breen, L. J. (2007). The researcher 'in the middle': Negotiating the insider/outsider dichotomy. The Australian Community Psychologist, 19(1), 163-174.

Bonner, A., \& Tolhurst, G. (2002). Insideroutsider perspectives of participant observation.Nurse Researcher, 9(4), and 7-19.

DeLyser, D. (2001). "Do you really live here?" Thoughts on insider research.Geographical Review, 91(1), 441-453. 
Gerrish, K. (1997). Being a 'marginal native': Dilemmas of the participant observer.Nurse Researcher, 5(1), and 25-34.

Geertz, C. (1973) The Interpretation of Cultures: Selected essays. New York: Basic Books.

Grey, C. (2005) A Fairly Short, Fairly Interesting and Reasonably Cheap Book About Studying Organisations. London: SAGE publications.

Hewitt-Taylor, J. (2002). Insider knowledge: Issues in insider research. Nursing Standard, 16(46), 33-35.

Hockey, J. (1993). Research methods: researching peers and familiar settings. Research Papers in Education, 8(2), and 199-225.

Labaree, R. V. (2002) The Risk of "Going Observationalist": Negotiating the hidden dilemmas of being an insider participant observer, Qualitiative Research, 2, 1, 97- 122.

Lesson, M. (2003). Methodology for close up studies - struggling with closeness and closure.Higher Education, 46, 167-193.

Lewis, D. K. (1973).Anthropology and colonialism.Current Anthropology, 14(5), 581602 .

Maruyama, M. (1981)Endogenous Research: Rationale. In P. Reason \& J. Rowan (eds) Human Inquiry: A sourcebook for new paradigm research. Chichester: John Wiley \& Sons pp. 227-238.
Merton, R. (1972) Insiders and Outsiders; A chapter in the sociology of knowledge, American Journal of Sociology, 78(July), 947.

Porteli, J. (2008). Researching a secondary school in Malta. In P. Sikes \& A. Potts (Eds.), Researching education from the inside (pp. 80-94). New York, NY: Rutledge.

Roberts, D. (2007) Ethnography and staying in your own nest. Nurse Researcher; 14: 3, 1524.

Rooney, P. (2005). Researching from the insidedoes it compromise validity? [Electronic Version].Level 3, May 2005, 1-19. Retrieved 12.12.13 from http:// level3.dit.ie/html/ issue3/ rooney/ rooney. Pdf.

Shaw, I. F. (2003).Ethics in qualitative research and evaluation.Journal of Social Work, 3(1), 9-29.

Smyth, A., \& Holian, R. (2008).Credibility Issues in Research from within Organisations. In P. Sikes \& A. Potts (Eds.), Researching education from the inside (pp. 33-47). New York, NY: Taylor \& Francis.

Tolson, d. et al (2006) constructing a new approach to developing evidence-based practice with nurses and older people.Worldviews on Evidence-Based Nursing; 3: 2, 62-72.

Wolff, K. H. (1950). The sociology of Georg Simmel. New York, NY: Free Press. 